\title{
Mesoscopic magnetoelectric effect in chaotic quantum dots
}

\author{
M. L. Polianski \\ Niels Bohr Institute, NBIA, Blegdamsvej 17, DK-2100 Copenhagen Denmark
}

(Dated: May 5, 2022)

\begin{abstract}
The magnitude of the inverse Faraday effect (IFE), a static magnetization due to an ac electric field, can be strongly increased in a mesoscopic sample, sensitive to time-reversal symmetry (TRS) breaking. Random rectification of ac voltages leads to a magnetization flux, which can be detected by an asymmetry of Hall resistances in a multi-terminal setup. In the absence of applied magnetic field through a chaotic quantum dot the IFE scale, quadratic in voltage, is found as an analytic function of the ac frequency, screening, and coupling to the contacts and floating probes, and numerically it does not show any effect of spin-orbit interaction. Our results qualitatively agree with a recent experiment on TRS-breaking in a six-terminal Hall cross.

PACS numbers: $73.23 .-\mathrm{b}, 73.63 . \mathrm{Kv}, 75.80 .+\mathrm{q}$
\end{abstract}

In pursuit of effects that combine spin and charge, great attention is paid to mutual effects of magnetic and electric fields. Their magnetoelectric manipulation is interesting not only scientifically, but also for possible applications. ${ }^{1}$ Obviously, any magnetooptic or magnetoelectric effect has its counterpart. For example, a ferromagnet polarizes the spins of electrons and conversely, a current can exert a torque on a magnetization vector and switch a magnetic domain in random access memory. ${ }^{2}$ Similarly, the Faraday effect, a rotation of the polarization of light by a magnetic field, has its inverse: a medium is magnetized by a beam of circularly polarized radiation. ${ }^{3}$ We consider the unusual properties of this magnetoelectric effect in small non-magnetic samples.

Classically, if a medium has a spatial inversion or timereversal symmetry (TRS), the expansion of its free energy $F$ does not have terms linear in $E$ or $H$, respectively. Magnetoelectric effects appear only due to mixing of $H$ and $E$, either as an $H_{i} E_{j}$ term if both symmetries are broken in equilibrium (magnetized anisotropic medium) or in a higher order " $H E E$ term" ${ }^{4} H_{i} E_{j} E_{k}^{(*)}$ if a strong electric field breaks TRS. In particular, this $H E E$ term leads to magnetization $M=\partial F / \partial H$, detectable in nonlinear effects like second-harmonic generation or rectification $^{5}$. The latter, known as inverse Faraday effect (IFE), is a static magnetization induced by perturbations at the frequency $\omega .{ }^{3,6}$ An electrical ac voltage, $V_{\omega}$, generated across a non-absorbing diffusive medium with mean-free time $\tau$ induces an asymmetry of the dielectric tensor. Since it is also linear in magnetic field, the classical IFE magnetization flux, $\varphi_{\mathrm{cl}}$, can be estimated, ${ }^{3}$

$$
\varphi_{\mathrm{cl}} \sim \alpha_{\mathrm{fs}} \Phi_{0}\left(e V_{\omega}\right)^{2} m v_{\mathrm{F}}^{3} /(\hbar \omega)^{3} c, \omega \tau \gg 1,
$$

where $\alpha_{\mathrm{fs}}$ is the fine structure constant, $\alpha_{\mathrm{fs}} \approx 1 / 137$, and $\Phi_{0} \equiv h / e$ is flux quantum. Importantly, this estimate could as well be obtained from the Joule heating and the asymmetry of the conductivity tensor. Since Eq. (1) contains small $v_{\mathrm{F}} / c$ and the fine-structure constant, one naturally asks: can we enhance the magnetic response to the $E$-field, which eventually breaks TRS?

To show that it is possible to exceed Eq. (1), we propose to use the sensitivity of electronic transport through a mesoscopic sample to the broken TRS (Ref 7 ) as a detector of magnetic flux. Indeed, disorder inevitably breaks a spatial symmetry in such a sample, and mesoscopic (sample-to-sample) fluctuations of transport occur on a flux scale $\Phi \lesssim \Phi_{0}$. TRS breaking could be induced either by a flux $\Phi$ of applied magnetic field, or by an IFE flux $\varphi$ created by additional ac perturbations. At $\Phi=0$, an indirect transport detection of $\varphi$ becomes possible in a multi-terminal setup with a separate pair of current- and voltage-probes ${ }^{8}$ measuring the Hall (or non-local) resistance $R_{H}$. Previously, Edelstein considered $R_{H} \propto V_{\omega}^{2}$ as a signature of IFE in a noncentrosymmetric diffusive twodimensional material with the mirror symmetry broken by spin-orbit interaction (SOI). ${ }^{9}$ Due to large spin-orbit scattering time, $\tau_{\text {so }} \gg \tau \gg 1 / \omega$, this effect is small, $\varphi_{\text {so }} \sim \Phi_{0}\left(e V_{\omega} / \varepsilon_{\mathrm{F}}\right)^{2}\left(\hbar / \omega \varepsilon_{\mathrm{F}} \tau_{\mathrm{so}}^{2}\right)$. In contrast, here we consider a large fluctuational effect.

Importantly, $R_{H} \neq 0$ even in small chaotic samples at zero field, but its random response to $\Phi$ can be used as a sample-specific gauge for the flux. Büttiker showed ${ }^{10}$ that the Onsager symmetry relations hold in mesoscopics, $R_{H}(\Phi)=R_{H}^{\mathrm{T}}(-\Phi)$, where ${ }^{\mathrm{T}}$ stands for the measurement with current- and voltage-probes exchanged; this prediction was confirmed in many linear transport experiments. ${ }^{11-13}$ Only recently did Chepelianskii and Bouchiat show that these relations are violated, when TRS is broken by additional ac perturbations. ${ }^{14}$ Since both applied $\Phi$ and induced $\varphi$ lead to the asymmetry $R_{H} \neq R_{H}^{\mathrm{T}}$, data in $R_{H}(0)-R_{H}^{\mathrm{T}}(0)$ suggest a shift of zero magnetic flux, i.e. an effective IFE flux $\varphi \neq 0$.

In this Rapid Communication we develop a theory that evaluates IFE in a multi-terminal chaotic quantum dot subjected to external ac perturbations at the frequency $\omega$ in the absence of applied magnetic field. First, we introduce a (sample-specific) gauge for magnetic flux, using the response of $R_{H}$ to small $\Phi$. Then we find the TRSbreaking in $R_{H}$ for the perturbed dot and evaluate the scale of fluctuations of the induced IFE flux $\varphi$ through its area:

$$
\varphi \sim \pm \Phi_{c}\left(e V_{\omega} / \varepsilon\right)^{2}, e V_{\omega} \ll \varepsilon=\max \left\{\hbar \omega, \hbar / \tau_{\mathrm{d}}, T\right\},
$$

where $\Phi_{c} \lesssim \Phi_{0}$ is the flux that completely breaks TRS, ${ }^{15}$ 
$\tau_{\mathrm{d}}$ is a typical dwell time of electrons in the dot, and $T$ is the temperature. Equation (2), the main result of our work, does not have definite sign, which is typical for quantum effects. Similarly to the equilibrium persistent current $^{16}$ or magnetic response of quantum dots, ${ }^{17,18}$ it does not contain $\alpha_{\mathrm{fs}}$ or large $\varepsilon_{\mathrm{F}}$. Experimentally, a sample can be magnetized not only by the flux $\Phi$ in equilibrium, but also by ac voltages at $\Phi=0$. Specific details of the sample rectify these voltages and lead to a random TRS breaking interpreted as an IFE magnetization. We expect this quantum interference effect to be measurable in any coherent sample of reduced dimensions. First we explain the model and major steps in the derivation of Eq. (2). Then we discuss how IFE is affected by screening, spin-orbit, and floating probes, and qualitatively compare our predictions to experiment. ${ }^{14}$

Model. We consider electronic transport through a multi-terminal $N$-channel chaotic quantum dot at the temperature $T$, see Fig. 1(a), and for simplicity assume the dot to be circular with radius $L$. Chaos is set due to either the diffusive motion of electrons, $l=\tau v_{\mathrm{F}} \ll L$, or their random scatterings from the boundaries, $L \ll l$, so that the ergodic time $\sim \max \{1, L / l\} L / v_{\mathrm{F}}$ needed to explore the dot is short. ${ }^{15,19}$ Therefore, electronic focusing and direct trajectories, present in some ballistic structures, ${ }^{7,14}$ are absent. Each of four contacts has $N_{\alpha} \gg 1$ ballistic orbital channels and is characterized by $n_{\alpha} \equiv N_{\alpha} / N$. Additional coupling (not necessarily ballistic) of floating probes ${ }^{20,21}$ is discussed in the end. ac voltages $V_{\alpha} \cos \left(\omega t+\phi_{\alpha}\right)$ applied at the same frequency $\omega$, but generally out of phase, are for convenience specified by the vectors $\vec{V}_{\alpha}=V_{\alpha} e^{i \phi_{\alpha}}$ in a complex plane [see Fig. 1(b)]. A top gate 0 with capacitance $C$ is biased by $\vec{V}_{0}$.

Screening in the dot is accounted for by the uniform time-dependent potential $U$, and its higher spatial harmonics are suppressed due to short ergodic time. ${ }^{19}$ This potential is found self-consistently from charge conser-

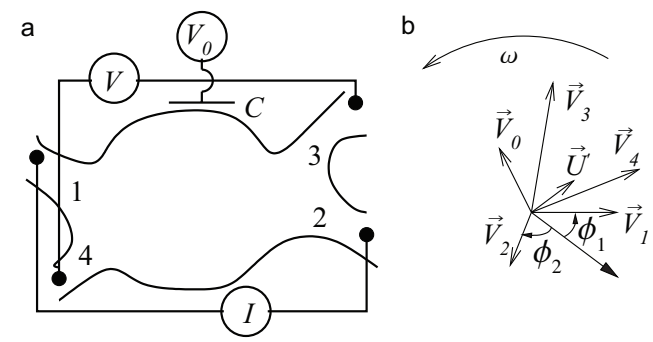

FIG. 1: (a) A gated chaotic dot in a four-terminal set-up, where the current through source-drain contacts 1 and 2 and the voltage drop between 3 and 4 yield $R_{H}=R_{12,34}$. IFE due to additional ac voltages $V_{\alpha} \cos \left(\omega t+\phi_{\alpha}\right)$ induces a random magnetization flux $\varphi$, detectable in $R_{H}-R_{H}^{\mathrm{T}}$; (b) Biases are specified by $\vec{V}_{\alpha}=V_{\alpha} e^{i \phi_{\alpha}}$ in the complex plane. IFE depends on the potential of the $\operatorname{dot}, \vec{U} \approx \vec{V}_{0}$ (or $\vec{U}^{\prime}$ ) for weak (or strong) interactions. vation and gauge invariance. ${ }^{22}$ The contributions leading to Coulomb blockade are small in $1 / N \ll 1$ and not taken into account. ${ }^{23}$ If $\Delta=2 \hbar^{2} / m L^{2}$ is the mean level spacing, the ratio $e^{2} / C \Delta$ defines the limits of weak/strong interaction. Using the dwell time of electrons, $\tau_{\mathrm{d}}=h / N \Delta$, we normalize the flux of applied magnetic field by the crossover flux that completely breaks TRS, $\phi=\Phi / \Phi_{c}$, where $\Phi_{c} / \Phi_{0}=2 L / \sqrt{\tau_{\mathrm{d}} v_{\mathrm{F}} l}, l \ll L$ $(l \rightarrow \pi L / 4$ if $L \ll l) .{ }^{15}$ We assume that the dot is not perturbed internally, so that the scattering is characterized by the energy-dependent scattering matrix $\mathcal{S}(\varepsilon)$. Statistical averages over ensemble, denoted by $\langle\ldots\rangle$, are found to leading order in $1 / N$ by diagrammatic technique in $\mathcal{S}(\varepsilon) .{ }^{24,25}$ We consider spinless electrons and normalize conductance/resistance by $\nu_{s} e^{2} / h$, where $\nu_{s}=2$ is spindegeneracy, and later compute the effect of SOI. ${ }^{26,27}$

The Hall resistance is measured as a linear response to additional voltages $\mathcal{V}_{\alpha}$ applied at small frequency $\Omega$ (experimentally, the frequency of lock-in $\Omega \lesssim 100 \mathrm{~Hz}$ ). If 1 and 2 are the source and drain, and the voltages are measured at 3 and 4 , one has $I_{1}=-I_{2}=I$ and $I_{3}=I_{4}=0$ defining $R_{H} \equiv R_{12,34}=\left(\mathcal{V}_{3}-\mathcal{V}_{4}\right) / I_{1}$ (Ref. 10) (applied voltages and current are related via $R_{x x}=\left(\mathcal{V}_{1}-\mathcal{V}_{2}\right) / I_{1} \approx\left(1 / n_{1}+1 / n_{2}\right) / N$, whose fluctuations might be neglected here). A transposition (exchange) of current- and voltage-probes, while keeping the same ac perturbations at $\omega$ and the applied magnetic flux, gives a different $R_{H}^{\mathrm{T}} \equiv R_{34,12}=\left(\mathcal{V}_{1}-\mathcal{V}_{2}\right) / I_{3}$. Due to randomness of the voltage drop $\mathcal{V}_{34(12)}$ across the dot, the resistance is more relevant than the conductance considered before. ${ }^{28}$

Derivation. For a non-interacting dot one can use scattering states ${ }^{29}$ (or Jauho-Meir-Wingreen formula ${ }^{30}$ ) to express the current in a contact $\alpha$ as a function of perturbations in a probe $\beta$. It responds not only linearly to $\mathcal{V}_{\beta \Omega}$, but also to $V_{\beta \omega}$, and for small $e V_{\omega} / \max \left\{\hbar \omega, T, \hbar / \tau_{\mathrm{d}}\right\} \ll$ 1 we expand current to first orders in this small parameter. This yields a conductance matrix $\tilde{g}_{\alpha \beta}$ at $\Omega \rightarrow 0$,

$$
\begin{aligned}
\tilde{g}_{\alpha \beta}= & \frac{\partial I_{\alpha}}{\partial \mathcal{V}_{\beta}} \approx-\int d \varepsilon \operatorname{tr}\left[\mathbb{1}_{\alpha} \mathcal{S}(\varepsilon) \mathbb{1}_{\beta} \mathcal{S}^{\dagger}(\varepsilon)-\mathbb{1}_{\alpha} \mathbb{1}_{\beta}\right] f^{\prime}(\varepsilon) \\
& +\left(\frac{e V_{\beta \omega}}{2}\right)^{2} \int d \varepsilon \frac{f(\varepsilon+h \omega)+f(\varepsilon-h \omega)-2 f(\varepsilon)}{(\hbar \omega)^{2}} \\
& \times \partial_{\varepsilon} \operatorname{tr} \mathbb{1}_{\alpha} \mathcal{S}(\varepsilon) \mathbb{1}_{\beta} \mathcal{S}^{\dagger}(\varepsilon) \equiv g_{\alpha \beta}+g_{\alpha \beta}^{\prime \prime}\left(e V_{\beta \omega} / 2\right)^{2} .
\end{aligned}
$$

While the first term is the usual dc conductance $g$, the second is an out-of-equilibrium contribution. It is similar, but not identical, to the photo-assisted current. ${ }^{29}$ However, Eq. (3) does not satisfy gauge-invariance: currents depend on $V_{\beta \omega}^{2}$ and change if all voltages are shifted by an arbitrary $\delta V_{\omega}$. Therefore, we consider the nearby gate and self-consistently find the internal potential of the dot $U(t)$ due to linear screening: a potential $U_{f}$ on some frequency $f$ is a linear combination of voltages at this frequency, $\vec{U}_{f}=\sum_{\beta} u_{\beta, f} \vec{V}_{\beta, f}$, where $u_{\beta, f}$ are complex characteristic potentials, which sum up to 1 . This potential depends on $\tau_{\mathrm{d}}$ and $\mathrm{RC}$ time $\tau_{\mathrm{RC}}=\tau_{\mathrm{d}} /\left(1+\nu_{s} e^{2} / C \Delta\right)$. ${ }^{22,31}$ The overall voltage shift by $-\vec{U}$ reduces the problem 
to the non-interacting, and now conductance, expressed via $F_{\gamma} \equiv e^{2}\left|\vec{V}_{\gamma}-\vec{U}\right|^{2} / 4$, reads as

$$
\tilde{g}_{\alpha \beta}=g_{\alpha \beta}+\sum_{\gamma=1}^{4} g_{\alpha \gamma}^{\prime \prime}\left(\delta_{\beta \gamma}-u_{\beta, 0}\right) F_{\gamma} .
$$

Since the sum of $\tilde{g}_{\alpha \beta}$ in Eq. (4) over $\alpha$ or $\beta$ vanishes, this sample-specific degenerate $\tilde{g}$ satisfies charge conservation and gauge invariance, respectively: unlike Eq. (3), it depends on differences $\vec{V}_{j}-\vec{V}_{k}$ unaffected by a voltage shift. Importantly, elements of $\tilde{g}$ and their correlators depend both explicitly on the static $u_{\beta, 0}$ and implicitly on dynamic $u_{\beta, \omega}$. The matrix $\tilde{g}_{2 \times 2} \propto 1-\sigma_{x}$ is always symmetric, ${ }^{32}$ but the TRS breaking becomes noticeable in a multi-terminal setup. Indeed, due to the symmetry to the matrix transposition, $g(\phi)=g^{T}(-\phi)$, the matrices $g(0)$ and $g^{\prime \prime}(0)$ are symmetric, but $\tilde{g}_{4 \times 4}$ is not, However, the measured result depends on the probe configuration.

Indeed, if some $i$-th probe does not draw any current, we can eliminate the $i$-th row and column from $\tilde{g}$ and simultaneously shift all voltages by $-\mathcal{V}_{i}$. We obtain $R_{H}$ inverting the remaining part $\tilde{g}_{3 \times 3}$, and the same method gives $R_{H}^{\mathrm{T}}$ in the other, transposed, configuration. When all $V_{\gamma \omega}=0$, the substitute $\tilde{g} \rightarrow g$ reproduces

$$
R_{H, \mathrm{dc}}=\frac{g_{31} g_{42}-g_{32} g_{41}}{\operatorname{det} g_{3 \times 3}}, R_{H, \mathrm{dc}}^{\mathrm{T}}=\frac{g_{13} g_{24}-g_{14} g_{23}}{\operatorname{det} g_{3 \times 3}},
$$

and the symmetry of $g$ immediately gives the Onsager relation, $R_{H, \mathrm{dc}}(\phi)=R_{H, \mathrm{dc}}^{\mathrm{T}}(-\phi) .{ }^{10}$ These Hall resistances, always equal at $\phi=0$, start to differ at weak magnetic flux $\phi \ll 1$, and to quantify their asymmetry we consider $\mathcal{A} \equiv\left(R_{H, \mathrm{dc}}-R_{H, \mathrm{dc}}^{\mathrm{T}}\right) / 2 \phi$. Its average vanishes, $\langle\mathcal{A}\rangle=0$, and the Gaussian fluctuations are given by

$$
\operatorname{Var} \mathcal{A} \equiv\left\langle\mathcal{A}^{2}\right\rangle-\langle\mathcal{A}\rangle^{2}=\frac{\left(n_{1}+n_{2}\right)\left(n_{3}+n_{4}\right)}{N^{4} n_{1} n_{2} n_{3} n_{4}} \int_{0}^{\infty} \mathcal{I} d \tau,(6)
$$

where $\mathcal{I} \equiv\left(4 / \tau_{\mathrm{d}}\right) e^{-\tau / \tau_{\mathrm{d}}}(\pi T \tau / \hbar)^{2} \sinh ^{-2}(\pi T \tau / \hbar)$. Compared to the classical Hall effect in such dots, this sensitivity is $\sim\left(L / N \lambda_{\mathrm{F}}\right)^{2} \gg 1$ stronger, but depends on the widths of the probes. As expected, when the dot is widely opened, $\Phi_{c} \rightarrow \Phi_{0}$ and Eq. (6) corresponds to the result of Ref. 28 up to a numerical coefficient $\sim 1$.

With additional ac perturbations the Hall resistances [Eq. (5)] gain IFE-corrections, and we find that $\delta R_{ \pm}=$ $\delta R_{H} \pm \delta R_{H}^{\mathrm{T}}$ are also normally distributed around zero, with

$$
\begin{aligned}
\operatorname{Var} \delta R_{ \pm}= & \frac{\left(n_{1}+n_{2}\right)\left(n_{3}+n_{4}\right)}{N^{4} n_{1} n_{2} n_{3} n_{4}} \int_{0}^{\infty} \mathcal{I} d \tau \frac{(1-\cos \omega \tau)^{2}}{(\hbar \omega)^{4}} \\
& \times\left(X_{12}+X_{34} \pm 2 e^{-4 \phi^{2} \tau / \tau_{\mathrm{d}}} Y_{12} Y_{34}\right), \quad(7) \\
\left\{\begin{array}{l}
X \\
Y
\end{array}\right\}_{\alpha \beta}= & \frac{n_{\alpha} n_{\beta}}{n_{\alpha}+n_{\beta}}\left\{\begin{array}{c}
F_{\alpha}^{2} / n_{\alpha}+F_{\beta}^{2} / n_{\beta}-\left(F_{\alpha}-F_{\beta}\right)^{2} \\
F_{\alpha} / n_{\alpha}+F_{\beta} / n_{\beta}
\end{array}\right\},
\end{aligned}
$$

Interestingly, the aforementioned dependence of $\tilde{g}$ and its fluctuations on $u_{\beta, 0}$ vanishes from Eq. (7) due to the antisymmetry of Eq. (5). Therefore, the statistics of $\delta R_{ \pm}$ is unaffected by the static characteristic potentials.

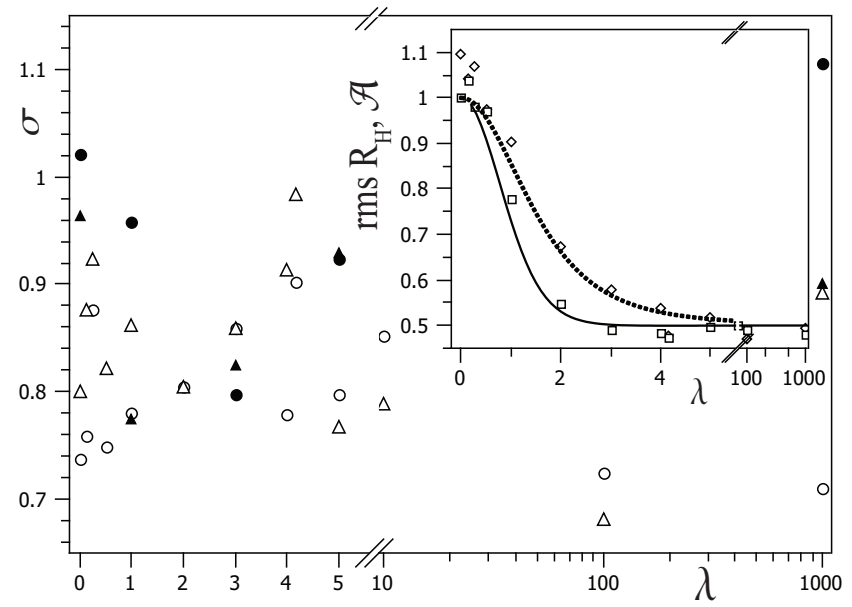

FIG. 2: The width $\sigma$ (०) of the IFE Lorentzian distribution and $\sigma_{\mathrm{m}}(\triangle)$ as functions of spin-orbit strength $\lambda$, normalized by $\sigma$ of Eq. (8); (inset) rms of $R_{H}(\diamond)[\mathcal{A}(\square)]$, normalized by $8[16] / N^{2}$, compared with theoretical dotted [solid] curves. Empty (filled) symbols correspond to $N=16(32)$.

The magnetization IFE flux can now be evaluated using the TRS breaking $\delta R_{-}$at $\phi=0, \varphi=\delta R_{-} / 2 \mathcal{A}$. To leading order, we can take $\mathcal{A}$ and $\delta R_{-}$uncorrelated and using Eqs. (6) and (7) find a Lorentzian mesoscopic distribution $P(\varphi)=\sigma /\left[\pi\left(\varphi^{2}+\sigma^{2}\right)\right]$ with

$$
\sigma^{2}=\frac{\int d \tau \mathcal{I}(1-\cos \omega \tau)^{2}}{4(\hbar \omega)^{4} \int d \tau \mathcal{I}}\left[X_{12}+X_{34}-2 Y_{12} Y_{34}\right] \Phi_{c}^{2} .
$$

The odd moments of $P(\varphi)$ vanish and the even ones diverge, but $\varphi$ is solely defined by $\sigma,\left\langle|\varphi / \sigma|^{ \pm 1 / 2}\right\rangle=\sqrt{2}$. In case one energy $\varepsilon$ among the energy scales $\hbar \omega, \hbar / \tau_{\mathrm{d}}, T$ is large compared to the other two, the ratio in Eq. (8) equals to $1 / \varepsilon^{4}$ multiplied by $3 / 8,3 / 2$ or $1 / 112$ respectively, which results in Eq. (2). The $\sigma^{2}$, a positivesemidefinite form of $F_{\gamma}$, can be created by a single $\vec{V}$ in some contact and vanishes only in a degenerate situation when all $F_{\gamma}$ are the same. One such example is when all voltages in the contacts have the same magnitude and phase and a gate-voltage $\vec{V}_{0} \neq \vec{V}_{\gamma}$ cannot induce IFE, see discussion of experiment. This situation is similar to a linearly polarized $\vec{E}$ not being able to induce the classical IFE, $\vec{M} \propto\left[\vec{E} \times \vec{E}^{*}\right]=0$. Unless this uniform regime is chosen to diminish $\varphi$, Eq. (2) remains a good order-of-magnitude estimate of IFE. As a function of frequency, $\sigma$ in Eq. (8) is modified by screening: in a strongly interacting dot, $\tau_{\mathrm{RC}} \ll \tau_{\mathrm{d}}$, in the high-frequency limit, $\omega \tau_{\mathrm{RC}} \gg 1$, the capacitor is short-cut compared to the contact resistances and $\vec{U} \approx \vec{V}_{0}$. As a result, IFE can occasionally become stronger than $1 / \omega^{2}$ due to the increased magnitude of $\left|\vec{V}_{\gamma}-\vec{U}\right|$ for the particular configuration of voltages [see Fig. 1(b)].

Until now, spin-orbit was neglected in the derivation of Eq. (8). To take it into account we construct a scattering matrix $\mathcal{S}(\varepsilon)$, which depends on magnetic field and 
SOI-strength, compute $g$ and $\tilde{g}$, and find IFE flux $\varphi=$ $\delta R_{-} / 2 \mathcal{A}$. For illustration, we take a simplified model ${ }^{27}$ of SOI using a parameter $\lambda \sim \hbar / \Delta \tau_{\text {so }}$ (Ref. 26) for the dot's random Hamiltonian $\mathcal{H}$ : in the limits $\lambda=0(\infty)$ it belongs to the Gaussian orthogonal (symplectic) Ensemble GOE, $\beta=1$ (GSE, $\beta=4$ ). In the crossover region the $2 M \times 2 M$ Hamiltonian with a fixed $\Delta$ is represented by $\mathcal{H}=\left[\sqrt{M} \mathcal{H}_{1}+(\lambda / 2) \mathcal{H}_{4}\right] / \sqrt{M+\lambda^{2}}$. Our numerics are done at $M=25 N$ to ensure $M \gg N$, for $T=0$, $\omega \ll \hbar / \tau_{\mathrm{d}}, N_{i}=N / 4$, and only $V_{1} \neq 0$. To find the IFE scale we fit $\sim 1200$ sample statistics to a shifted Lorentzian, and the result is presented in Fig. 2 together with $\sigma$ given by moments, $\sigma_{\mathrm{m}}=\left\langle|\varphi|^{1 / 2}\right\rangle /\left\langle|\varphi|^{-1 / 2}\right\rangle$. Deviations of $\sigma(\lambda)$ from Eq. (8) are attributed to a relatively small number of channels and appear to be nonsystematic. The inset in Fig. 2 shows rms of $R_{H}$ and $\mathcal{A}$ from the fits to normal distributions in very good agreement with our predictions $0.5\left(1+3 /\left(1+4 \lambda^{2} / N\right)^{2}\right)^{1 / 2}$ and $0.5\left(1+\left(3-4 \lambda^{2} / N\right) /\left(1+4 \lambda^{2} / N\right)^{4}\right)^{1 / 2}$, respectively. The lack of any systematic trend of the available statistics in the main plot (compared to the inset) suggests that the mesoscopic IFE is unaffected by SOI, in contrast with the weak IFE existing only due to SOI. ${ }^{9}$

The generalization from a 4-contact setup to $M$ contacts includes arbitrarily coupled floating contacts, which are typical in a Hall measurement. The voltage in a floating probe is adjusted to allow exchange of electrons with the dot, but not to draw any current. As a result, our analytical results become renormalized using $N=\sum_{i=1}^{4} N_{i}+N_{f}$. Not necessarily integer, $N_{f}$ is defined similarly to $N_{i}$ by the total dimensionless conductance $N_{f}$ of all available floating contacts. In the dephasing probe model ${ }^{20}$ the inelastic scattering with time $\tau_{\phi}$ adds $h / \Delta \tau_{\phi}$ fictitious channels to $N_{f}$. This redefinition decreases $\tau_{\mathrm{d}}$ and $n_{\alpha}$ used in $\mathcal{I}$ and Eqs. (6)-(8).

Discussion of experiment. Reference 14 measured a TRS breaking at $\phi=0$ in gated ballistic Hall samples, where each of $6 \quad(=4+2$ floating $)$ contacts had $N_{\alpha} \sim 200$ channels. AC perturbations were applied at $\omega \sim 10^{6}-10^{10} \mathrm{~Hz}$, either asymmetrically (sample A) or uniformly (sample B). Corrections to $R_{H}$, quadratic in small perturbation amplitude, were measured as functions of $\omega$ in the sample A. They vanish for $\omega<\omega_{0}$ and for $\omega>\omega_{c}$ become of the same order, and a single-parameter pumping in a billiard was used to numerically reproduce non-monotonic $R_{H}-R_{H}^{\mathrm{T}}$ as a function of $\omega$. On the contrast, sample B showed no TRS breaking, $\delta R_{H} \approx \delta R_{H}^{\mathrm{T}}$.

Alternatively, data can be interpreted as mesoscopic fluctuations of IFE in a chaotic sample. They are visible even at large $N$ due to vanishing classical effect. Unfortunately, data can not be directly compared with RMT result (7) because the Hall cross does allow direct trajectories resulting in much smaller non-local voltages and at $0.3 \mathrm{~K}$ it is beyond the universal regime.Experiment interprets $\omega_{0}$ as a frequency characteristic for (unknown) capacitive coupling with the contacts, when ac voltages become noticeable. In our setup the voltages are given and this circuit effect is not accounted for. At low frequencies $\delta R_{ \pm}$vary $\propto\left(\omega \tau_{\mathrm{d}}\right)^{2}$, but the anti-symmetric component is generally smaller by construction [cf. \pm in Eq. (7)]. Beyond the threshold $\omega_{c} \sim 1 / \tau_{\mathrm{d}}$ both fluctuate similarly with a typical period $\Delta \omega \sim 1 / \tau_{\mathrm{d}}$. Indeed, in the experiment $\delta R_{+}$is usually larger and $\omega_{c} \sim \Delta \omega$ is consistent with $\tau_{\mathrm{d}} \approx 3$ ps expected from a dot with the sample area and $N \approx 1200$. For the uniformly perturbed sample $\mathrm{B}$ with equal voltages $\vec{V}_{1 \ldots .}$, Eq. (7) indeed results in fluctuations of $\delta R_{+}$and $\delta R_{-}=0$ observed in experiment, which does not enter a high frequency regime. However, it is desirable to perform measurement in a truly chaotic sample without direct trajectories and at lower $T$ and $N$ to increase quantum fluctuations, or measure IFE directly using superconducting quantum interference device, similarly to persistent current. ${ }^{16}$

Conclusions. We consider a time-reversal symmetry breaking by external ac voltages. Rectified perturbations generate a static magnetoelectric effect, a random magnetization of a mesoscopic sample, similar to the classical Inverse Faraday Effect. Mesoscopic fluctuations of the magnetization flux can be measured using out-ofequilibrium transport in a multi-terminal quantum dot. We estimate a typical flux, quadratic in voltages, as a function of frequency, screening, coupling to reservoirs, and numerically find that spin-orbit scattering has a very small effect on IFE. Our results allow qualitative comparison with and explain most important features of a recent experiment in TRS breaking.

Acknowledgements. Alexei Chepelianskii suggested this problem to me, and I thank him, Hélène Bouchiat and Markus Büttiker for discussions and comments.
1 M. Fiebig, J. Phys. D 38, R123 (2005).

2 S. Maekawa, Concepts in Spin Electronics (Oxford University Press, USA, 2006).

3 L. Landau and E. Lifshitz, Electrodynamics of Continuous Media (Pergamon Press, New York, 1960).

${ }^{4}$ H. Schmid, Ferroelectrics 161, 1 (1994).

${ }^{5}$ M. Fiebig, V. V. Pavlov, and R. V. Pisarev, J. Opt. Soc. Am. B 22, 96 (2005).

${ }^{6}$ R. Hertel, J. Magn. Magn. Mater. 303, L1 (2006).

7 C. Beenakker and H. van Houten, Solid State Phys. 44, 1
(1991).

8 L. J. van der Pauw, Philips Res. Rep. 13, 1 (1958).

${ }^{9}$ V. M. Edelstein, Phys. Rev. Lett. 95, 156602 (2005).

10 M. Büttiker, Phys. Rev. Lett. 57, 1761 (1986).

11 A. D. Benoit, S. Washburn, C. P. Umbach, R. B. Laibowitz, and R. A. Webb, Phys. Rev. Lett. 57, 1765 (1986).

12 S. Washburn and R. A. Webb, Rep. Prog. Phys. 55, 1311 (1992).

13 K. L. Shepard, M. L. Roukes, and B. P. van der Gaag, Phys. Rev. B 46, 9648 (1992). 
14 A. D. Chepelianskii and H. Bouchiat, Physical Review Letters 102, 086810 (2009).

15 C. W. J. Beenakker, Rev. Mod. Phys. 69, 731 (1997).

16 L. P. Lévy, G. Dolan, J. Dunsmuir, and H. Bouchiat, Phys. Rev. Lett. 64, 2074 (1990).

17 B. L. Altshuler, Y. Gefen, Y. Imry, and G. Montambaux, Phys. Rev. B 47, 10335 (1993).

18 K. A. Matveev, L. I. Glazman, and A. I. Larkin, Phys. Rev. Lett. 85, 2789 (2000).

19 I. L. Aleiner, P. W. Brouwer, and L. I. Glazman, Phys. Rep. 358, 309 (2002).

20 M. Büttiker, Phys. Rev. B 33, 3020 (1986).

${ }^{21}$ P. W. Brouwer and C. W. J. Beenakker, Physical Review B 55, 4695 (1997).

22 M. Büttiker, A. Prêtre, and H. Thomas, Phys. Rev. Lett. 70, 4114 (1993).

${ }^{23}$ P. W. Brouwer, A. Lamacraft, and K. Flensberg, Physical Review B 72, 075316 (2005).

${ }^{24}$ P. W. Brouwer and C. W. J. Beenakker, J. Math. Phys.
37, 4904 (1996).

25 M. L. Polianski and P. W. Brouwer, J. Phys. A 36, 3215 (2003).

26 J.-H. Cremers, P. W. Brouwer, and V. I. Falko, Phys. Rev. B 68, 125329 (2003).

27 S. Adam, M. L. Polianski, X. Waintal, and P. W. Brouwer, Phys. Rev. B 66, 195412 (2002).

28 M. Ma and P. A. Lee, Phys. Rev. B 35, 1448 (1987).

29 M. H. Pedersen and M. Büttiker, Phys. Rev. B 58, 12993 (1998).

30 A.-P. Jauho, N. S. Wingreen, and Y. Meir, Phys. Rev. B 50, 5528 (1994).

31 M. Büttiker and M. L. Polianski, J. Phys. A 38, 10559 (2005).

32 IFE measured in a 2-terminal setup using the position of the weak localization peak does not survive mesoscopic averaging, and a sample-specific result is smeared out by $\mathrm{UCF}$. 